\title{
Fetal Cerebellar Vermis Circumference Measured by 2-Dimensional Ultrasound Scan: Reference Range, Feasibility and Reproducibility
}

Authors

Affiliations

\author{
M. Spinelli ${ }^{1}$, C. Sica ${ }^{2}$, L. D. Meglio' ${ }^{2}$, D. Bolla ${ }^{3}$, L. Raio ${ }^{3}$, D. Surbek
}

${ }^{1}$ Department of Clinical Research, Prenatal Medicine, Inselspital Bern Universitatsklinik fur Frauenheilkunde, Bern, Switzerland 2 Obstetrics and Gynecology, Private Centre "Diagnostica ecografica e prenatale Aniello Di Meglio s.r.l.", Naples, Italy ${ }^{3} \mathrm{OB} / \mathrm{GYN}$, University of Bern, Bern, Switzerland

${ }^{4}$ OB \& GYN, Inselspital, Bern, Switzerland

\section{Key words}

- prenatal diagnosis

- ultrasound 2D

- cerebellar vermis

received $\quad 12.02 .2016$

accepted $\quad 04.10 .2016$

Bibliography

DOI http://dx.doi.org/

10.1055/s-0042-119952

Ultrasound International Open

2016; 2: E124-E128

(c) Georg Thieme Verlag KG

Stuttgart · New York

ISSN 2199-7152

Correspondence

Dr. Marialuigia Spinelli, MD, OB/GYN specialist, PhD fellow

Department of Clinical

Research Prenatal Medicine

Inselspital Bern Universitatsk-

linik fur Frauenheilkunde

Effingerstraße 102

3010 Bern

Switzerland

Tel.: +41/762/773513

marialuigiaspinelli@live.it

\section{License terms}

\begin{abstract}
$\nabla$

Purpose: To provide 2-dimensional ultrasonographic (2D-US) normograms of cerebellar vermis biometry, as well as to evaluate the feasibility and the reproducibility of these measurements in clinical practice.

Materials and Methods: A prospective crosssectional study of 328 normal singleton pregnancies between 18 and 33 weeks of gestation. Measurements of the fetal cerebellar vermis circumference (VC) in the mid-sagittal plane were performed by both a senior and a junior operator using 2D-US. VC as a function of gestational age (GA) was expressed by regression equations. In 24 fetuses 3-dimensional (3D) reconstructed planes were obtained in order to allow comparisons with 2D-US measurements. The agreement between 2D and 3D measurements and the interobserver variability were assessed by interclass correlation coefficients (ICC).
\end{abstract}

\section{Introduction \\ $\nabla$}

Recent knowledge in neurophysiology has shown that the cerebellar vermis is a fundamental midline structure which is involved not only in proprioception and synergy of movements, but also in language, behavior and cognitive development [1-4].

Currently, imaging of the fetal posterior fossa represents an integral part of sonographic screening for fetal anomalies [5]. However, the standard antenatal evaluation of these structures is limited to the axial plane and does not provide the assessment of cerebellar vermis size and integrity, which are thought to address the differential diagnosis of a wide range of pathologic conditions, including both minor and major anomalies [6-9].
Results: Satisfactory vermis measurements could be obtained in $89.9 \%$ of cases. The VC (constant $=-12.21 ;$ slope $=2.447 ; \mathrm{r}=0.887, \mathrm{p}<0.0001$ ) correlated linearly with GA. A high degree of consistency was observed between $2 \mathrm{D}$ and 3D ultrasound measurements (ICC $=0.84695 \% \mathrm{CI}$ 679-0.930) as well as between measurements obtained by different examiners (ICC $=0.89095 \%$ CI 989-0.945).

Conclusion: 2-dimensional ultrasonographic measurements of cerebellar vermis throughout gestation in the mid-sagittal view seem to be feasible and reproducible enough to be potentially used in clinical practice. Such measurements may supply a tool for accurate identification of posterior fossa anomalies, providing the basis for proper counseling and management and of these conditions.
For this reason, a wrong/missed diagnosis of vermian dysgenesis, in particular of the dorsal part, is still possible. Furthermore, there is disagreement on issues regarding the prenatal diagnosis of Dandy-Walker complex and the corresponding autopsy findings [10].

A window for assessing cerebellar vermis size, integrity and relationship with other nearby brain structures can be provided by the midsagittal view of the fetal brain, as recently shown by other authors $[11,12]$.

However, at present, only minimal data on the antenatal measurements of the vermis are available [10-12], mainly performed using 3-dimensional (3D) technology.

The aim of this paper is to measure the cerebellar vermis circumference (VC) in normal fetuses during 
routine prenatal 2D-US examination, through the mid-sagittal plane, throughout pregnancy, in order to:

A. provide 2-dimensional (2D-US) nomograms of the cerebellar VC based on a large number of normal fetuses;

B. evaluate the reproducibility of these measurements between different operators (senior vs. junior);

C. evaluate the correlation among 2D and 3D-US measurements;

D. provide a reference model in order to address diagnosis of vermian anomalies.

\section{Materials and Methods \\ $\nabla$}

We performed a prospective cross-sectional study of sonographic imaging of the fetal cerebellar vermis. Included were lowrisk pregnant women with well-established dates [determined by a well-defined last menstrual period and confirmed by measurement of the crown-rump length (CRL) on first-trimester US], and singleton, non-anomalous fetuses. All participants had a negative history for systemic diseases, as well as intact fetal membranes, normal amniotic fluid volume, and were not in labor at the time of inclusion in the study. Indications for ultrasound examination were assessment of either fetal anatomy or fetal growth.

Gestational age (GA) ranged between 14 and 36 weeks. Fractions of weeks were computed to the nearest week, with fractions of $\leq 4$ days and $>5$ days assigned to the lower and higher weeks, respectively.

All deliveries occurred after 37 completed weeks. The neonates were healthy, and no infants had evidence of growth disturbances (fetal growth restriction or macrosomia). All women in the study delivered in 4 referral hospitals and underwent examination by an attending pediatrician.

As in cross-sectional studies, each fetus was considered only once. The study was approved by our Institutional Review Board and all women gave written informed consent to participate in the study.

The ultrasound machine used for 2D-US were standard Aloka (Aloka Co., Ltd, Tokyo, Japan) and Voluson E10 (GE Healthcare Ultrasound, Milwaukee, WI, USA) equipped with a curved linear array transabdominal transducer $(2-5 \mathrm{MHz})$ as well as with a transvaginal 4-8 $\mathrm{MHz}$ probe.

The fetal vermis was examined in the mid-sagittal plane, with demonstration of the corpus callosum, the cervical spine and the cisterna magna. Freeze-frame ultrasound capabilities and electronic on-screen calipers were used for the measurements of the vermis circumference (VC). Color Doppler imaging was not utilized.

The transvaginal approach was reserved for when the mid-sagittal view of the fetal brain could not be obtained by the transabdominal route due to fetal position.

Every measurement was taken online during the $20-40 \mathrm{~min}$ allocated for the routine scan and detected twice with the mean calculated.

In 33 fetuses each measurement was repeated twice by 2 blinded examiners (C.S. and M.S.) in order to assess the reproducibility of the measurements. The 2 operators were identified as n. 1 (senior, i.e. more than 5 years of experience and expertise with prenatal ultrasound) and n.2 (junior, i.e. less than 5 years of experience with prenatal ultrasound), and all measurements were numbered as 1 when performed by the senior or 2 for the junior operator.

In 24 fetuses a mid-sagittal view of the fetal head was also obtained by 3-dimensional (3D) reconstructed planes in order to allow comparisons with measurements obtained by 2D-US.

Transabdominal 3D volume acquisitions were performed on the same ultrasound machine used for 2D scans, equipped with a 4-8 $\mathrm{MHz}$ transabdominal probe, using the technique previously reported by other authors $[10,13]$.

Brain volumes were acquired starting with the obtainment of a transcerebellar axial view of the fetal brain during fetal rest and maternal rest using a transabdominal acquisition angle of $45-60^{\circ}$ depending on the GA.

\section{Statistical analysis}

Statistical analysis was performed with GraphPad Prism version 5.00 for Windows, (GraphPad Software, San Diego CA) and SPSS statistical software (version 19.0; SPSS Inc., Chicago, IL, USA).

To generate the VC reference intervals, only cases between 18 and 33 weeks of gestation were included in the analysis, as previously reported [10-12]. The reference ranges for the VC were constructed using the method previously described by Royston and Wright [14]. Polynomial regression analysis was performed to identify the regression curves that best fitted the mean and standard deviation (SD) of the VC as a function of gestational age. The standard deviation scores ( $Z$ scores) were calculated using the formula: observed VC measurement - mean VC/SD. To assess the model fit, the Gaussian distribution of the $\mathrm{Z}$ scores was checked using the Kolmogorov-Smirnov test. $10^{\text {th }}$ and $90^{\text {th }}$ percentiles for the cross-sectional VC throughout gestation were obtained as previously described using the formulas: mean \pm $1.645 \mathrm{SD}$, and mean $\pm 1.28 \mathrm{SD}$, respectively [14].

The agreement between 2D and 3D measurements, transabdominal and transvaginal scans, as well as the interobserver variability were assessed by interclass correlation coefficients (ICC). Bland-Altman plots were used too.

Statistical significance was considered achieved when P was less than 0.05 .

\section{Results \\ $\nabla$}

Consecutive pregnant women meeting the eligibility criteria $(n=397)$ were initially enrolled in the study. An adequate vermis measurement was obtained in $89.9 \%$ of these cases $(n=357)$, using either the transabdominal $(n=325)$ or the transvaginal $(\mathrm{n}=32)$ route.

In the excluded 40 cases, a mid-sagittal view of the fetal brain on 2D-US could not be obtained mainly due to unfavorable fetal position or excessive fetal movement.

10 of the included pregnant women were lost at follow-up. Thus, 347 cases were ultimately considered for our analysis.

- Fig. 1a and $\mathbf{b}$ demonstrate a mid-sagittal view of the fetal head obtained by transabdominal 2D-US and 3D reconstructed planes, respectively, showing the VC and adjacent anatomical landmarks at 20 and 24 weeks of GA, respectively, with electronic calipers denoting measurements.

- Table 1 shows the clinical characteristics of the study population including perinatal outcome data.

To generate the VC reference intervals, 328 fetuses, ranging from 18 to 33 weeks, were utilized. The regression equation for the mean VC (y) according to gestational age $(\mathrm{x})$ was: $\mathrm{y}=-12.21+2.447 \mathrm{x}$ and 

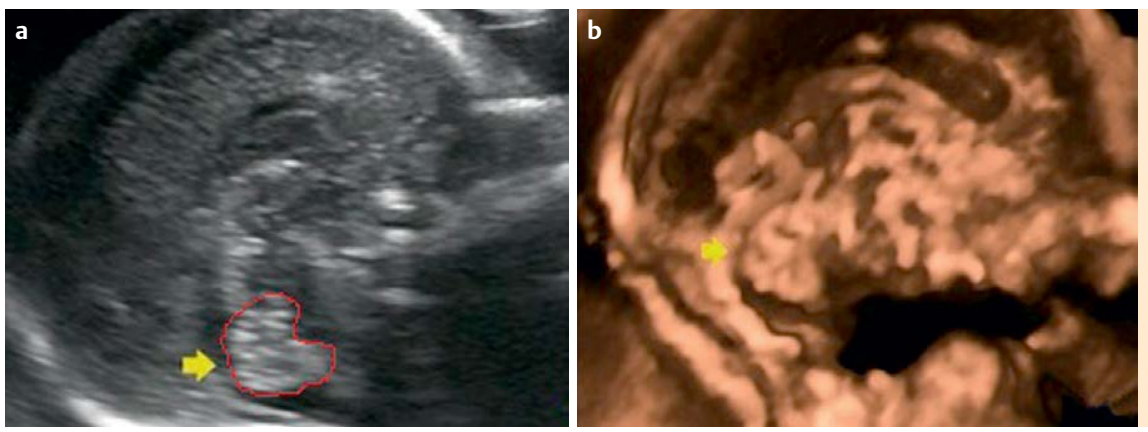

Fig. 1 Ultrasound images showing the cerebellar vermis (yellow arrow) obtained by $2 \mathrm{D}$ ultrasound at 22 weeks of gestation a and 3D ultrasound at 24 weeks of gestation $\mathbf{b}$ Vermis circumference (VC) is outlined in red $\mathbf{a}$.

Table 1 Clinical Characteristics of the Study Population Including Perinatal Outcome Data.

\begin{tabular}{|ll}
\hline Characteristic & Value \\
\hline Age, $y$ & 31 (range: $21-39$ ) \\
\hline Body mass index, $\mathrm{kg} / \mathrm{m}^{2}$ & $23.5 \pm 3.38$ \\
\hline Gravidity & $1.4 \pm 0.61$ \\
\hline Parity & $1.0 \pm 0.41$ \\
\hline Birth weight, $\mathrm{g}$ & $3084 \pm 503$ \\
\hline Birth percentiles & $45 \pm 12$ \\
\hline Male/female ratio & 0.59 \\
\hline Data are presented as mean \pm SD where applicable
\end{tabular}

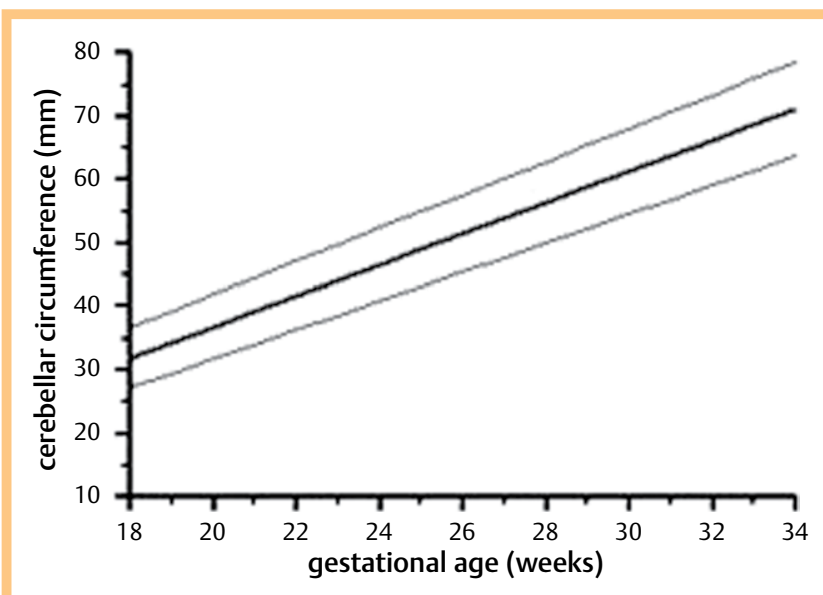

Fig. 2 Plot showing the cerebellar circumference observed measurements and the fitted $10^{\text {th }}, 50^{\text {th }}$, and $90^{\text {th }}$ percentiles for gestational age.

Table 2 Predicted cerebellar vermis circumference (VC) ( $\mathrm{mm}$ ) by gestational age (GA).

\begin{tabular}{lllll}
$\mathbf{G A}$ & $\mathbf{n o .}$ of & $\mathbf{1 0}^{\text {th }}$ & $\mathbf{5 0}^{\text {th }}$ & $\mathbf{9 0}^{\text {th }}$ \\
& cases & percentile & percentile & percentile \\
\hline 18 & 18 & 27.77 & 32.40 & 37.03 \\
19 & 24 & 29.86 & 33.96 & 38.50 \\
\hline 20 & 70 & 32.47 & 37.93 & 42.39 \\
\hline 21 & 75 & 34.85 & 38.75 & 44.65 \\
\hline $22-23$ & 54 & 37.75 & 43.02 & 47.29 \\
\hline $24-25$ & 24 & 42.72 & 47.42 & 53.12 \\
\hline $26-27$ & 19 & 46.44 & 52.1 & 59.76 \\
\hline $28-30$ & 23 & 53.30 & 59.75 & 65.20 \\
\hline $31-33$ & 21 & 59.87 & 65.15 & 73.83 \\
\hline
\end{tabular}

for the standard deviation ( $\left.\mathrm{y}^{\prime}\right)$, it was y' $=1.348+0.1302 \mathrm{x}$. $\bullet$ Fig. 2 and $\triangle$ Table 2 show the VC observed measurements and the fitted $10^{\text {th }}, 50^{\text {th }}$, and $90^{\text {th }}$ percentiles for gestational age.
A high degree of consistency was observed between 2D and 3D ultrasound measurements of the cerebellar vermis in the series of 24 fetuses studied with both techniques (interclass correlation coefficient $(\mathrm{ICC})=0.84695 \%$ confidence interval $(\mathrm{CI}) 0.679$ 0.930 ), as well as between transvaginal and transabdominal scans in the series of 32 cases approached transvaginally (interclass correlation coefficient $($ ICC $)=0.87495 \%$ confidence interval (CI) 0.746-0.976).

When the interobserver variability was assessed for measurements obtained on 2D-US in the series of 33 fetuses, the ICC was 0.890 and its $95 \%$ confidence interval was $0.989-0.945$.

Bland-Altman plots show the mean differences and 95\% limits of agreement between 2D and 3D (mean difference $=-0.604$ $95 \% \mathrm{CI}-1.499-0.292$ ) ( $\bullet$ Fig. 3a) as well as the mean difference of interobserver agreement (mean difference $=0.94895 \%$ CI -0.046-1.943) (॰ Fig. 3b).

At birth, all infants underwent a detailed examination by a pediatrician or neonatologist and all were found to be normal.

\section{Discussion}

$\nabla$

Cerebellar vermis hypoplasia can be segmental, and its recognition may be difficult on prenatal US. Furthermore, an enlarged fourth ventricle or cistern magna may cause the displacement or the rotation of the normal vermis, which could be wrongly considered as hypoplastic $[15,16]$.

Assessment of the integrity and size of the cerebellar vermis is fundamental in evaluating posterior fossa abnormalities since it represents the key feature of these malformations. Thus, the precise assessment of vermis biometry is extremely important in order to correctly distinguish between physiological and pathological size and to detect also slight, segmental anomalies; such defects may be associated with other structural malformations, syndromes and chromosomal or genetic diseases, as well as with cognitive, language and behavioral dysfunction among children [10,17-22].

As a consequence, imaging of the cerebellar vermis has been suggested as an integral part of the routine ultrasonographic study of the fetal brain [10].

At present, the standard antenatal evaluation of the cerebellum is limited to the axial plane, which does not provide a window to provide cerebellar vermis biometry as well as the relationship with cerebellar hemispheres and the other structures of the posterior fossa. This can be obtained only by a mid-sagittal view of the fetal brain.

For this reason, recent research has been focused on the ultrasonographic study of the fetal brain through the mid-sagittal plane, which is becoming a fundamental step for the study of midline brain anomalies [10-13,23]. 

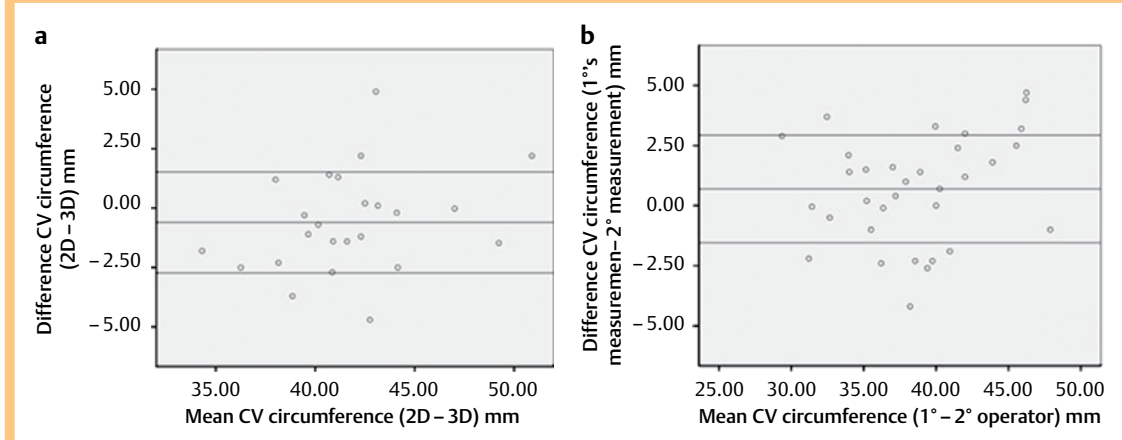

Fig. 3 Bland-Altman plots of the paired measurement obtained by 2D and 3D ultrasound $\mathbf{a}$ and by two different examiners $\mathbf{b}$.
Recently, other authors have demonstrated the advantages of 3D technology, which enables the acquisition of a volume of the fetal brain in a transverse section, to reconstruct the mid-sagittal view $[12,13,24]$.

However, although interest in the ultrasonographic study of the posterior fossa is increasing, only few authors assess vermis biometry in clinical practice when 3D technology is not available, and data regarding the normal biometry of the cerebellar vermis are still minimal. Therefore, a wrong/missed diagnosis of vermis abnormality, in particular when it is segmental, is still probable in US evaluation [18,25-27].

The present study is based on the need for a major increase in the use of such 2D-US measurements in order to evaluate the feasibility and reproducibility of vermian biometry in clinical practice.

The choice to start our measurements from the $18^{\text {th }}$ week of pregnancy is due to the fact that the cerebellar vermis is not yet fully developed prior to this week, as explained in the literature [10-12].

Our data, in accordance with previous papers, confirm that the cerebellar vermis grows in a linear fashion throughout gestation and the growth pattern correlates well with GA [10$12,23,27,28]$.

To our knowledge, only a few papers have measured VC for assessing vermian growth throughout gestation. Our nomograms constructed by 2D-US correlated well with those previously reported by Malinger et al. [27] and Lei et al. [28]. We could not correlate our nomograms with those provided in other papers [10-12], since they focused on vermian diameter and area but not on VC.

We focused exclusively on VC because this parameter is rarely reported in the literature compared with vermis diameter. Furthermore, we believe that the assessment of the circumference can provide precise information about the morpho-biometry of the vermis, is easy to perform and may provide more guarantee of agreement between different operators.

All these values can serve as a reference for all sonographers evaluating the posterior fossa.

To our knowledge, this study includes the largest series $(\mathrm{n}=328)$ ever published for the assessment of cerebellar vermis growth throughout gestation in a 2D transabdominal manner. The series of Zalel et al. [10] included a small number of patients, while the series of Malinger et al. [28] focused exclusively on the 2D transvaginal approach. Vinals et al. [12], Rizzo et al. [11], and Lei [28] constructed cerebellar vermis biometric reference curves with the aid of 3D technology.

Despite the large number of fetuses included in our analysis, we observed an inhomogeneous distribution of cases among the dif- ferent gestational weeks, with most of the measurements performed before 24 weeks. This may be due to the fact that the major number of controls in normal pregnancies is scheduled in the second trimester and should be considered as a limit of our study. We did not observe any gender difference in the measurement of the VC. To our knowledge, this data is in accordance with previous papers which do not mention gender differences in their measurements [10-12,28].

In our study, we performed most of the US scans using the transabdominal approach. Indeed, we preferred the routine transabdominal rather than the transvaginal route, since the latter may be less immediate and more time-consuming, as well as less tolerated by pregnant women. However, in 32 cases a transvaginal probe was necessary for obtaining an adequate vermis view due to fetal position. We observed high agreement between values when transvaginal measurements were compared with transabdominal ones at the respective gestational age. These latter data encourage us to continue to prefer the transabdominal route when possible.

In contrast to other authors, we focused on obtaining the midsagittal plane via 2D-US rather than 3D-US. Although 3D technology can be very useful in many cases, especially those of unfavorable fetal position, it is an expensive tool, requires an expert operator and is not readily available in some clinical contexts. We believe that the assessment of vermis biometry should be encouraged and used among different centers, even when 3D technology is not accessible.

In our study, we reported good reproducibility between $2 \mathrm{D}$ and 3D measurements. These data are in agreement with other reports [10] and suggest that $2 \mathrm{D}$ measurements can be as adequate and accurate as $3 \mathrm{D}$ measurements for the intrauterine study of some structures of the fetal brain.

To the best of our knowledge, this is the first time that interobserver variability between a senior and a junior operator has been tested. It is of interest that the reliability of the measurements taken by the 2 examiners with varying skill levels was quite good as expressed by the interclass correlation coefficient (ICC) of nearly 0.90 .

This last finding may support the hypothesis that these measurements may be sufficiently feasible and reproducible to be assessed more frequently as well as applied in more clinical contexts.

\section{Conclusion \\ $\nabla$}

Our study shows how cerebellar vermis biometry can be obtained in a feasible and reproducible manner on transabdominal 2D US from 18 to 33 weeks of gestation with good agreement 
with 3D measurements. Our data support the use of the transabdominal 2D route to assess such biometry and encourage operators to perform these measurements.

The nomograms developed in this study and the few previous studies should enable accurate evaluation of the cerebellar vermis and may facilitate the identification of cases with suspected vermian anomalies, as well as help with the differential diagnosis of posterior fossa anomalies, providing the basis for proper management and counseling for these conditions.

\section{References}

1 Barkovich AJ. Developmental disorders of the midbrain and hindbrain. Front Neuroanat 2012; 6: 1-10

2 Rapoport M, van Reekum R, Mayberg $H$. The role of the cerebellum in cognition and behavior: A selective review. J Neuropsychiatry Clin Neurosci 2000; 12: 193-198

3 Shevell M, Majnemer A. Clinical features of developmental disability associated with cerebellar hypoplasia. Pediatr Neurol 1996; 15: 337-339

4 Courchesne E, Yeung-Courchesne R, Press GA, Hesselink JR, Jernigan TL. Hypoplasia of cerebellar vermis lobules VI and VII in autism. N Engl J Med 1988; 318: 1349-1354

5 American Institute of Ultrasound in Medicine. AIUM practice guidelines for the performance of an antepartum obstetric ultrasound examination. J Ultrasound Med 2010; 29: 157-166

6 Salomon LJ, Alfirevic Z, Berghella V, Bilardo C, Hernandez-Handrade E, Johnsen SL, Kalache K, Leung KY, Malinger G, Munoz H, Prefumo F, Toi A, Lee W. Practice guidelines for performance of the routine midtrimester fetal ultrasound scan. Ultrasound Obstet Gynecol 2010; 37: $116-120$

7 International Society of Ultrasound in Obstetrics \& Gynecology Education Committee. Sonographic examination of the fetal central nervous system: Guidelines for performing the basic examination and the fetal neurosonogram. Ultrasound Obstet Gynecol 2007; 29: 109-116

8 Joubert MEJ, Eisenring JJ, Robb JP, Andermann F. Familial agenesis of the cerebellar vermis. A syndrome of episodic hyperpnea, abnormal eye movements, ataxia and retardation. Neurology 1969; 19: 813-825

9 Bordarier C, Aicardi J. Dandy-Walker syndrome and agenesis of the cerebellar vermis: diagnostic problems and genetic counselling. Dev Med Child Neurol 1990; 32: 285-294

10 Zalel Y, Seidman D.S, Brandt N. et al. The development of the fetal vermis: an in utero sonographic evaluation. Ultrasound Obstet Gynecol 2002; 19: 136-139

11 Rizzo G, Pietrolucci M.E, Mammarella S et al. Assessment of cerebellar vermis biometry at $18-32$ weeks of gestation by three-dimensional ultrasound examination. The Journal of Maternal-Fetal and Neonatal Medicine 2012; 25: 519-522
12 Viñals $F$, Muñoz $M$, Naveas $R$ et al. The fetal cerebellar vermis: anatomy and biometric assessment using volume contrast imaging in the C-plane (VCI-C). Ultrasound Obstet Gynecol. 2005; 26: 622-627

13 Rizzo G, Pietrolucci M.E, Capponi A. Assessment of corpus callosum biometric measurements at 18-32 weeks gestation by 3-dimensional sonography. J Ultrasound Med 2011; 30: 47-53

14 Royston P, Wright EM. How to construct 'normal ranges' for fetal variables. Ultrasound Obstet Gynecol. 1998; 11: 30-38

15 Robinson AJ. Inferior vermianhypoplasia - preconception, misconception. Ultrasound Obstet Gynecol 2014; 43: 123-136

16 Sgaier SK, Millet S, Villaneuva MP et al. Morphogenetic and cellular movements that shape the mouse cerebellum; insight from genetic fate mapping. Neuron 2005; 45: 27-40

17 Sherer DM, Sokolovski M, Dalloul M et al. Nomograms of the axial fetal cerebellar hemisphere circumference and area throughout gestation. Ultrasound Obstet Gynecol 2007; 29: 32-37

18 Kapur RP, Mahony BS, Finch L et al. Normal and abnormal anatomy of the cerebellar vermis in midgestational human fetuses. Birth Defects Res A Clin Mol Teratol 2009; 85: 700-709

19 Dekain LH, Kanel H, El-Bashir HO. Joubert syndrome labeled as hypotonic cerebral palsy. Neurosciences (Riyadh) 2014; 19: 233-235

20 Poretti A, Boltshauser E, Doherty D. Cerebellar hypoplasia: differential diagnosis and diagnostic approach. Am J Med Genet Semin Med Genet 2014; 166C: 211-226

21 Barzegar M, Malaki M, Sadegi-Hokmabadi E. Joubert syndrome with variable features: presentation of two cases. Iran J Child Neurol 2013; 7: $43-46$

22 Basson MA, Wingate RJ. Congenital hypoplasia of the cerebellum: developmental causes and behavioral consequences. Front Neuroanat 2013; 3: 1-16

23 Ginath S, Lerman-Sagie T, Haratz Krajden $K$ et al. The fetal vermis, pons and brainstem: normal longitudinal development as shown by dedicated neurosonography. J Matern Fetal Neonatal Med 2013; 26: 757-762

24 Tonni G, Grisolia G, Sepulveda W. Second trimester fetal neurosonography: reconstructing cerebral midline anatomy and anomalies using a novel three-dimensional ultrasound technique. Prenatal Diagnosis. 2014; 34: 75-83

25 Carroli SG, Porter H, Abdel-Fattah S et al. Correlation of prenatal ultrasound diagnosis and pathological findings in fetal brain anomalies. Ultrasound Obstet Gynecol. 2000; 16: 149-153

26 Pilu G, Visentin A, Valeri B. The Dandy-Walker complex and fetal sonography. Ultrasound Obstet. Gynecol. 2000; 16: 115-117

27 Malinger G, Ginath S, Sagie-Lerman T et al. the fetal cerebellar vermis: normal development as shown by transvaginal ultrasound. Prenatal Diagnosis 2001; 21: 687-692

28 Lei T, Xie HN, Zhu YX, Zheng J, Zhang F, Feng JL. Date-independent parameters: an innovative method to assess fetal cerebellar vermis. Cerebellum 2015; 14: 231-239 